\title{
22
}

\section{Jovian seismology}

\section{BENOîT MOSSER}

Institut d'Astrophysique de Paris, 98bis, bd Arago, 75014 Paris, France ; MOSSER@IAP.FR

\begin{abstract}
This paper reviews a new astrophysical subject: seismology of the giant planets. Seismology is dedicated to the sounding of the interior structure of any object; on the other hand, the interiors of the Jovian planets need to be constrained, in order to improve our knowledge of their structure and of their evolution, as well as the thermodynamical laws involved at high pres sures and low temperatures. The relationship between Jovian seismology and, first, Jovian internal structure, and second, high pressure physics, is examined, in order to determine the task of "dioseismology" $\dagger$ in the next years. We present then the seismological theoretical approaches developped since the pionnering work of Vorontsov et al. (1976), who calculated the frequencies of the Jovian eigenmodes. We report the first observational attempts for the detection of the oscillations of Jupiter. We discuss the observational results and examine what can be done in the future.
\end{abstract}

La sismologie des planètes géantes apparaît comme un centre d'intérêt astrophysique d'avenir. Elle doit permettre en effet - et il s'agit en fait du seul outil dont l'on dispose - de sonder les intérieurs de ces planètes, actuellement mal connus, mais dont la détermination représente un intérêt majeur. Cet article récapitule aussi bien les diverses approches théoriques développées depuis l'article précurseur de Vorontsov et al. (1976) que les di-

† This neologism, constructed in the same manner as the substantive helioseismology, should represent the seismology of all four giant, or Jovian, planets. 
verses expériences menées pour détecter les oscillations de la planète Jupiter. L'accent est mis sur les liens reliant l'étude sismologique des planètes géantes avec d'une part leur structure interne, d'autre part la physique hyperbare gérant les équations d'état utilisées pour décrire le comportement de l'enveloppe fluide. Il apparaît que les deux problématiques, que l'on souhaiterait distinctes, s'enchevêtrent à l'envi. L'enjeu de la "diosismologie" consiste en parvenir, essentiellement par un développement dans un premier temps des observations, à démèler l'imbroglio actuel. Des pistes sont proposées, qui permettent d'établir, à partir de résultats sismologiques, des résultats univoques en terme de structure interne.

\subsection{Introduction}

\subsubsection{The interior structure of giant planets}

The image we have from any astrophysical object is two-dimensional. What we see from the giant planets is in fact a very thin layer from where the photons escape or are reflected. In Jupiter for instance, the upper atmosphere is sounded by spectroscopy from a few microbars down to the 10-bar level. Some physical data, as pressure, temperature or density, cannot be measured in the deep interior. However, the values of the mass, the gravitational moments $J_{2}, J_{4}$ and $J_{6}$, the rotation period of the planetary core and the luminosity constrain interior models. But the density profiles obtained from these integral quantities are strongly non unique. In addition, the following points must be noted:

- The giant planet interiors correspond to pressure and density ranges where the equation of state (EOS) of hydrogen, helium and heavier elements are very far from the perfect gas law (Chabrier, these proceedings). The determination of the pressure-density profiles in the planetary interior would be a unique tool for determining the EOS of a hydrogen-helium gas mixing at very high pressure.

- The precise determination of the actual state of the giant planet interior is a clue for their former evolution.

- The measurement of the concentrations of helium or other elements in the whole planet and not only in the upper atmosphere, as well as the determination of the structure discontinuities in all four giant planets are key points for planetology. 


\subsubsection{Seismology}

Seismology is a very powerful tool for the investigation of the interior structure of any object. A simple seismological experience consists of sounding a wall by knocking it: the sound it makes helps determining its consistency. In the same way, we need to "listen" to the Jovian resonances in order to determine of what the planetary interior is made. Because of its fluid interior, Jupiter looks like a star or the Sun, and the Jovian seismological study is a priori very similar to the one of any spherical fluid object. The acoustic modes (e.g. sound waves) which are favored in a sphere are expressed very crudely by:

$$
\nu_{n, \ell} \propto\left[n+\frac{\ell}{2}\right] \nu_{0}
$$

where $n$ is the radial order of the mode. The degree $\ell$ is related to the first index of the spherical harmonics $Y_{\ell}^{m}$ associated to the mode, and $\nu_{0}$ is the acoustic characteristic frequency. The pattern described by Eqt. 22.1 is approximately followed, for example, by the solar modes, the one of $\alpha$-Cen (Pottasch et al. 1992) as well as Procyon (Gelly et al. 1986) and the Jovian modes (Schmider et al. 1991, Mosser et al. 1993). The signature of each object appears in the smaller terms which are not expressed by Eqt. 22.1. The calculation, measurement and interpretation of these terms are the task of dioseismology.

\subsubsection{Historical review}

The first paper about the seismology of giant planets is from Vorontsov, Zharkov \& Lubimov (1976). The authors present the two basic ideas of dioseismology: the measurements of the oscillation periods is a unique tool for investigating the planetary interiors; intensive energetics can lead to the excitation of such oscillations. Low degree and low order pressure modes of Jupiter and Saturn are calculated, as well as discontinuity modes due to the core. This paper was followed by four other articles (Vorontsov \& Zharkov 1981; Vorontsov 1981, 1984a and b), which represent a very complete approach of the specific problems of Jovian seismology. Structure discontinuities, oblateness and differential rotation are considered as perturbations of a spherical and continuous state. Bercovici \& Schubert (1987) have introduced a more simple approach which is based on the ray tracing theory. They propose some possible excitation mechanisms for the modes, and are the first to give an estimate of the Jovian mode amplitude, expressed by the observable velocity in the troposphere: about $0.5 \mathrm{~m} . \mathrm{s}^{-1}$. The first 
Jovian échelle diagrams (see Table 22.7) are due to Mosser et al. (1988) and Vorontsov et al. (1989); they show how strong is the influence of the core on the oscillation pattern.

The first attempt for detecting the Jovian oscillations came 13 years after the first theoretical paper. It is due to Deming et al. (1989). The long delay between the first theoretical development of Jovian seismology and the first observations is surprising, when considering the importance of the subject as well as the efforts which have been made at the same time in helioseismology. It can be understood only because of the real difficulty of the observation. In order to achieve the necessary resolution, the detection has to be made, with a large telescope, continuously over several nights, and therefore needs a stable detector. The first tentative detection was negative. Due to the geometry of the IR detector, only "high" degree modes $(\ell>10)$ with azimuthal order $|m|=\ell \nmid$ were searched. According to the conditions required for the detection of Jovian pulsations (Schmider et al. 1991, Mosser 1993), it seems that the observation conditions were not favorable.

\subsubsection{Summary}

A review on planetary seismology by Lognonné \& Mosser (1993) has presented general theoretical and observational results on Jovian seismology. In this review, we propose a more detailed approach of the interconnection of the giant planets seismology and their interior structure. The main questions concerning the interior of the giant planets and their seismological consequences are presented in Section 22.2. Seismological calculations and observations of pressure modes are developped in Section 22.3. Finally, the discussion proposed in Section 22.4 gives clues to what can be in the future the efficiency of seismology for disentangling the planetary interiors. We will restrict our attention principally to Jupiter, the only giant planet whose oscillations have been likely detected. There is no conceptual difference between Saturn and Jupiter. The case of Uranus and Neptune is somewhat different, since they have a greater core. Their theoretical oscillations spectra are depicted in Section 22.3. But, due to their low luminosity, it will be a long time before they can be observed.

† Such degrees cannot be considered as high in helioseismology, since the Sun appears 40 times greater than Jupiter in the $8 \mathrm{ky}$ ! 


\subsection{Giant planets structure}

The aim of this chapter is twofold. It wants first to establish the link between the construction of giant planets models and high pressure physics; secondly between the models and seismology. In order to examine these two points, we will focus on the method currently used for the construction of the giant planets interior models.

\subsubsection{Current constraints}

\subsubsection{The gravitational moments}

The current parameters constraining the structure are the gravitational moments $J_{2 n}$ (Table 22.1). These parameters express the decomposition of the non spherical gravitational potential of the planet. $J_{0}$ is simply the mass of the planet. $J_{2}, J_{4}$ and $J_{6}$ of Jupiter have been measured by the Voyager spacecrafts (Campbell \& Synnot 1985). $J_{2}$ indicates the presence of a more dense core, which is supposed to be made of heavy materials, ices and rocks (Hubbard \& Marley 1989). The uncertainty on $J_{6}$ is too high to permit any constraint.

\subsubsection{Convection and composition}

The internal flux radiated by Jupiter is supposed to be transported everywhere in the planet by convection (Hubbard 1968). The radiative opacity of hydrogen and helium is too high to allow energy to be carried out by radiation. The presence of convection implies that the planet is adiabatically stratified (in fact, a very little of superadiabaticity is needed to evacuate the internal energy, but at such a low level that it is usually neglected). Jovian models are then supposed to be fully adiabatic. Since an adiabatic temperature profile has been measured under the 1-bar pressure level by Voyager 2 (Lindal et al. 1981), the adiabat starts at the 1-bar pressure level, with a tropospheric composition as given by the observations (Gautier \& Owen 1989), and a temperature of $165 \mathrm{~K}$. Models follow the same adiabat from the 1-bar level to the center of the planet. The gradient in the core is surely not adiabatic, since the planetary flux goes to zero at the center. Furthermore, the adiabat surely changes with the composition gradients. However, the EOS of the heavy materials used in the core, extraplolated from lower pressure theoretical estimations (Hubbard \& Marley 1989) are also assumed to be adiabatic. 


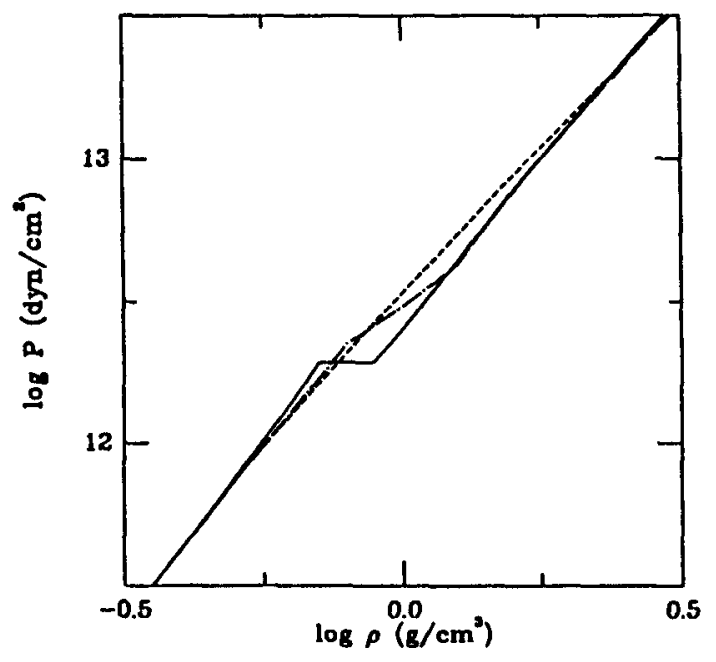

Fig. 22.1 Pressure-density relation along a pure hydrogen adiabats (from Chabrier et al. 1992, Fig. 2). Differences between the EOS (solid curve: Saumon et al. EOS with plasma phase transition (PPT), dashed curve: without PPT (interploation), dot-dashed: Marley \& Hubbard EOS) are very small, except in the vicinity of the PPT.

\subsubsection{The plasma phase transition (PPT)}

The influence of the plasma phase transition of hydrogen (PPT) on the Jovian structure has been presented by Chabrier et al. (1992) and Saumon et al. (1992). The unique role of the PPT in the Jovian thermal balance, if really a first order transition, must be emphasized. Even if, according to Saumon et al. (1992), the actual latent heat release contributes to only about $1 \%$ of the radiated power, the hypothetical transformation of all the metallic hydrogen into molecular hydrogen should provide the actual Jovian flux during not less than $10^{10}$ years! The role of the latent heat in the planetary evolution has to be taken very carefully into account.

\subsubsection{Saturn, Uranus and Neptune}

Standard models of Saturn do not differ qualitatively from Jovian models. Because of the lower mass, the PPT occurs deeper in the planet. The low helium abundance measured in the troposphere is explained by the unmiscibility of helium in the metallic region, hence its depletion in the envelope. The pressure in the fluid envelope of Uranus and Neptune is not high enough to permit PPT. These two giant planets present a large ice shell surrounding a rock core, and a thin fluid envelope (Fig. 22.2, Table 22.3). 


\subsubsection{Construction of the interior models}

The models take into account the oblate shape of the planet (see Table 22.1) due to the high rotation velocity. To be consistent, the calculations must be two-dimensionally developped. Variables are functions of the inner radius $r$ and of the colatitude $\theta$ (axisymmetry is assumed). The theory of figures, as exposed in Zharkov \& Trubitsyn (1978), shows how it is possible to reduce, through some coefficients describing the oblate shape of the planet, the twodimensional dependence to a pure radial dependence. All models assume a solid body rotation, with a rotation period equal to that of the magnetic field (system III). Then, two equations govern the evolution of density and pressure:

$$
\left\{\begin{aligned}
\text { hydrostatic equilibrium : } & \frac{\mathrm{d} p}{\mathrm{~d} r}=-\rho g \\
\text { EOS } \equiv \text { adiabat : } & \rho=\rho(p)
\end{aligned}\right.
$$

The gravitational field $g$ expresses simply in function of the density: $g=$ $4 \pi G \int_{0}^{r} \rho u^{2} \mathrm{~d} u / r^{2}$. The hydrostatic equilibrium equation carries no essential information, but the interior is fluid. What governs the model is the EOS which is used. The Jovian adiabat results $\rho(p)$ from the ideal additive volume law:

$$
\frac{1}{\rho(p)}=\frac{\mathrm{X}}{\rho(p)_{\mathrm{X}}}+\frac{\mathrm{Y}}{\rho(p)_{\mathrm{Y}}}+\frac{\mathrm{Z}}{\rho(p)_{\mathrm{Z}}}
$$

where $\mathrm{X}, \mathrm{Y}$ and $\mathrm{Z}$ are respectively the mass fraction of hydrogen, helium and heavier elements; $\rho(p)_{i}$ is the EOS of species $i$ following the planetary adiabat. $\mathrm{Y}$ and $\mathrm{Z}$ in the outer envelope are in agreement with the observational results, whereas their mean value is inferred from the solar composition $\left(\bar{Y}_{\text {Jup }}=\mathrm{Y}_{\odot}\right.$, enrichment in $\mathrm{Z}$, cf. Table 22.2). This implies a composition discontinuity, whose exact location remains undetermined (Gudkova et al. 1989, Zharkov \& Gudkova 1991, 1992).

Fig. 22.1 represents three pure hydrogen adiabats, which have been used for the construction of Jovian standard models (Fig. 22.2). It can be remarked that the difference in the pressure-density relations (as high as $20 \%$ at the location of the PPT) leads to difference in the density profiles (Fig. 22.3) less than $2 \%$, except at the core level. The main characteristics of a Jovian standard model (Table 22.3) are finally:

- the rotation and the resulting oblateness of the planet.

- the core, with a discontinuity constrast of about 4 with respect to the fluid envelope. 
Table 22.1. Jupiter: primary constraints

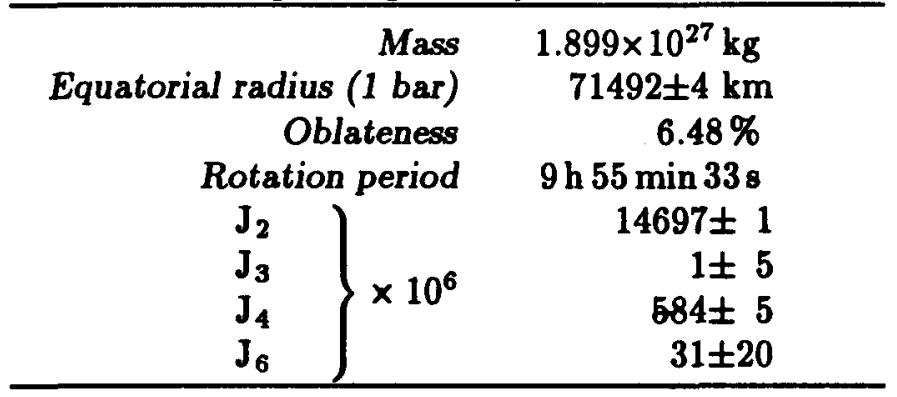

Table 22.2. Jupiter: composition and structure envelope $\quad Y \simeq 0.20$ and $Z \simeq 0.02$; adiabatic gradient

$P P T$ transition $\mathrm{H}_{2}-\mathrm{H}_{\text {metal }}$ around the $1.2 \mathrm{Mbar}$ level core rocks and ices, mixed or separated

Table 22.3. Giant planet models

\begin{tabular}{|c|c|c|c|}
\hline Model & Authors & Core & Envelope \\
\hline $\left.\begin{array}{l}\text { JUP } 1 \\
\text { JUP2 } \\
\text { JUP4 } \\
\text { JUP5 } \\
\text { JUP6 } \\
\text { JUP7 }\end{array}\right\}$ & $\begin{array}{l}\text { Hubbard \& Marley } 1989 \\
\text { Chabrier \& Saumon } 1992 \\
\text { Gudkova \& Zharkov } 1989\end{array}$ & $\begin{array}{l}\text { mixed ice and rock } \\
\text { rock core }+ \text { ice shell } \\
\text { mixed ice and rock }\end{array}$ & $\begin{array}{l}\Delta Z \neq 0 \\
\text { no PPT } \\
\text { PPT } \\
\text { PPT, } \Delta Y \neq 0 \\
\Delta Z \neq 0\end{array}$ \\
\hline $\begin{array}{l}\text { SAT1 } \\
\text { URA1 } \\
\text { NEP1 }\end{array}$ & $\begin{array}{l}\text { Hubbard \& Marley } 1989 \\
\text { Hubbard \& Marley } 1989 \\
\text { Podolak } 1991\end{array}$ & $\begin{array}{l}\text { rock core }+ \text { ice shell } \\
\text { rock core }+ \text { ice shell }\end{array}$ & \\
\hline
\end{tabular}

- the transition from molecular to metallic hydrogen, occuring around the 1.2 Mbar level in the Jovian interior.

- convection everywhere in the planet.

Finally, in the standard model frame, the use of a given EOS, associated with a given set of secondary hypothesis, corresponds to a given interior model (Table 22.3). Uniqueness of the model is not insured, so that new constraints, stronger than the gravitational constraints, are needed. 


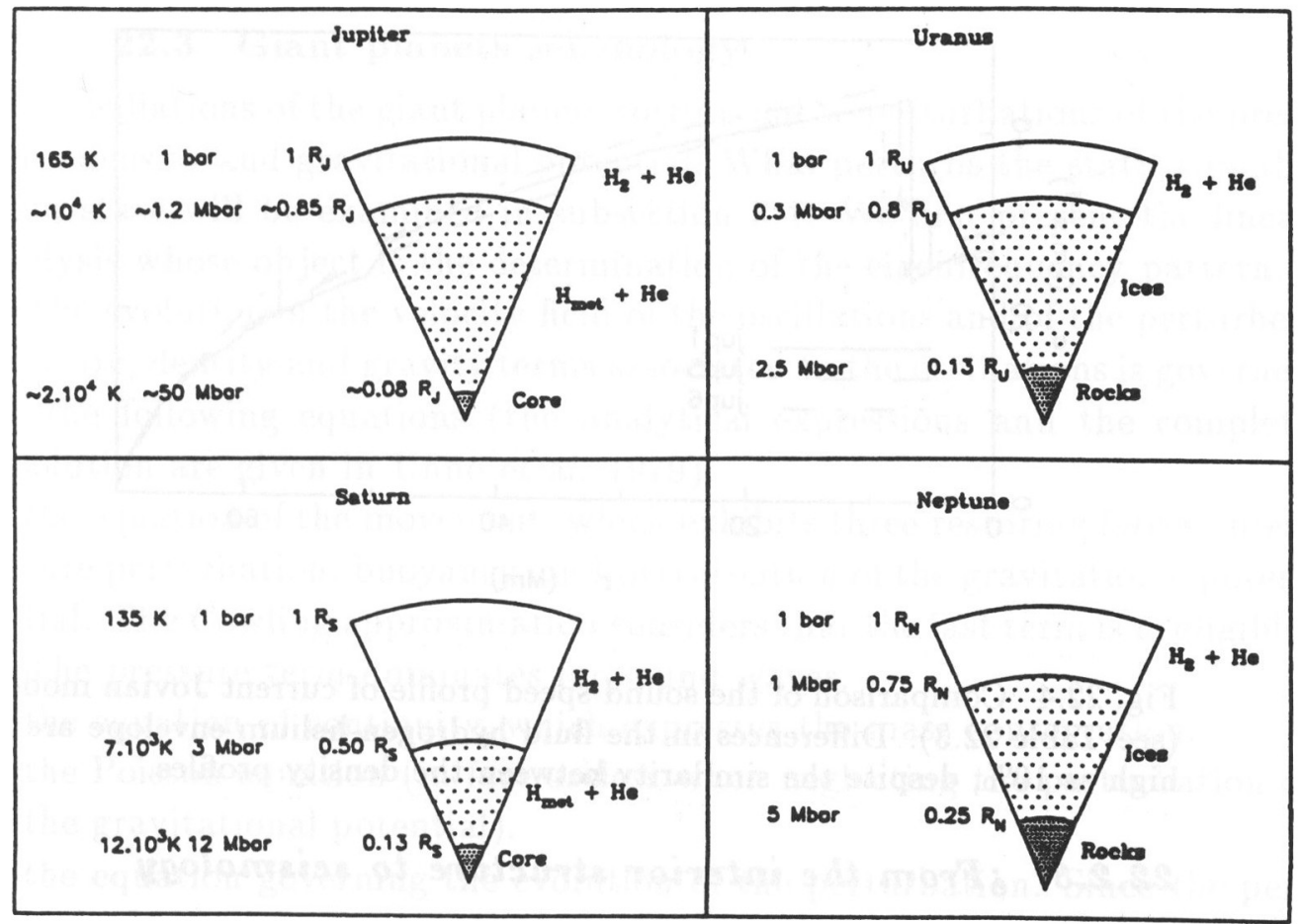

Fig. 22.2 Standard model of the four giant planets. Models of Jupiter and Saturn have three layers: the core, and the two fluid envelopes. The pressure in the fluid envelope of Uranus and Neptune is not high enough to permit the transition to metallic hydrogen. The greatest density contrast is at the rock core for Jupiter and Saturn, and the ice core for Uranus and Neptune.

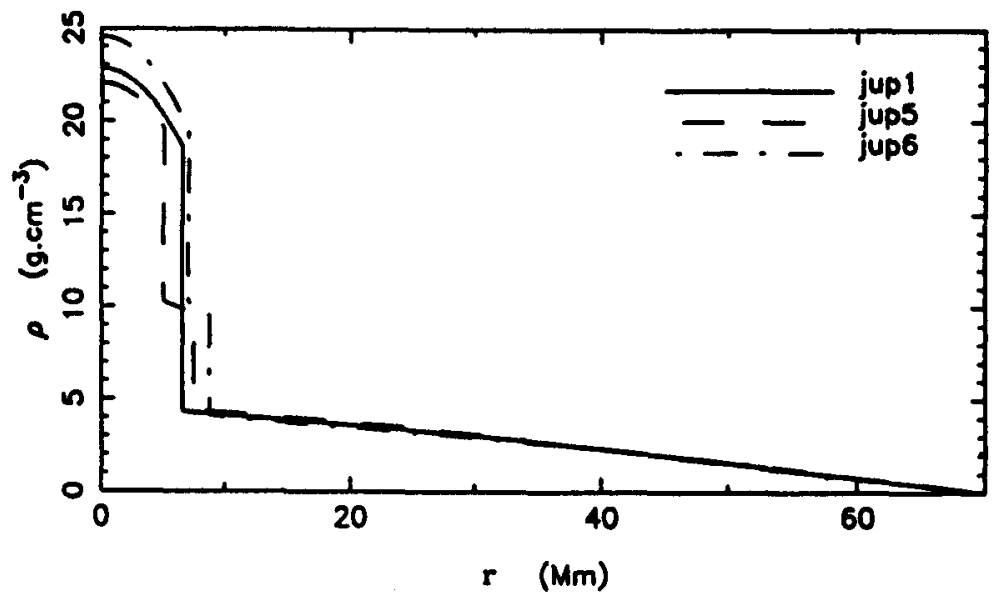

Fig. 22.3 Comparison of the density profile of current Jovian models (see Table 22.3). Differences in the fluid hydrogen-helium envelope are not perceptible, but the core structures are very different. 


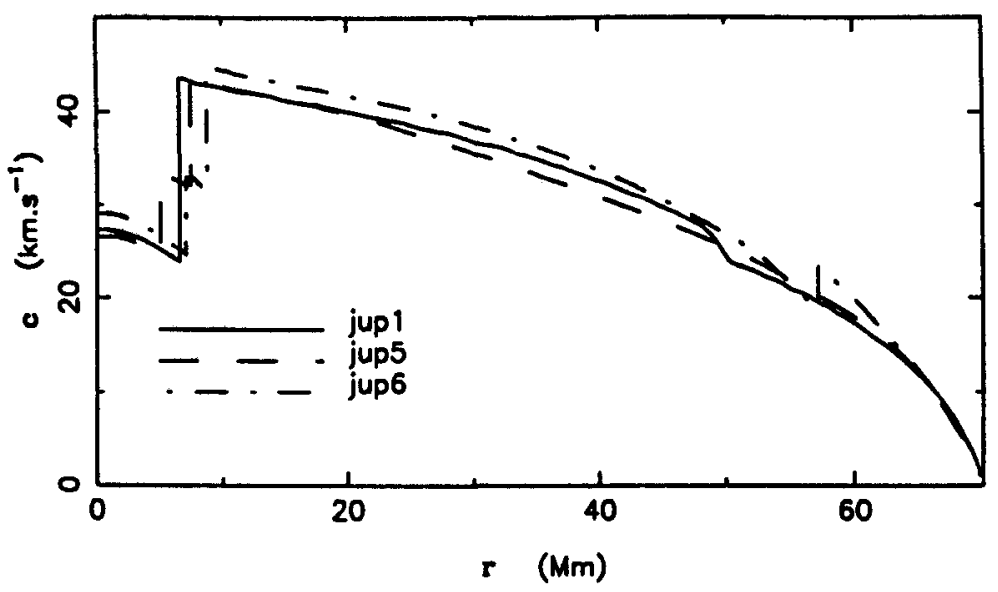

Fig. 22.4 Comparison of the sound speed profile of current Jovian models (see Table 22.3). Differences in the fluid hydrogen-helium envelope are as high as $10 \%$, despite the similarity between the density profiles.

\subsection{3 \&From the interior structure to seismology}

An important parameter for studying pressure oscillations is the sound speed. Its expression is easily derived from the interior structure:

$$
c^{2} \stackrel{\text { def }}{=}\left(\frac{\partial p}{\partial \rho}\right)_{\mathrm{S}} \stackrel{\text { adiabaticity }}{\equiv} \frac{\mathrm{d} p_{0}}{\mathrm{~d} \rho_{0}}
$$

where $p$ and $\rho$ represent the pressure and density perturbation of the wave and $p_{0}$ and $\rho_{0}$ the non perturbed terms. The definition of the sound speed - a second order derivative of the free energy - first shows the capability of seismology to distinguish between different EOS. In fact, even if different adiabatic EOS used for Jupiter give very similar density profiles in the fluid envelope (Fig. 22.3), they lead to sound speed profiles that differ by about $10 \%$ (Fig. 22.4). Secondly, the calculation of the sound speed puts in evidence the importance of the hypothesis of adiabaticity. A non adiabatic gradient would lead to a different sound speed profile.

Finally, one must emphasize the influence of the core. The high density constrast at the core frontier induces a strong sound speed contrast, which drastically affects the oscillations pattern (Section 22.3.). Furthermore, according to the manner the models are constructed, the core plays a very important role: its mass, even if very small in current models (about $1 \%$ of the mass of Jupiter) is adjusted so that the mass of the model fits the planetary mass. Therefore, the core mass and size - and therefore its seismological signature - depend crucially on the EOS used in the fluid envelope. 


\subsection{Giant planets seismology}

The oscillations of the giant planets correspond to perturbations of the pressure, density and gravitational potential. What perturbs the static equilibrium state will be examined in subsection 3.4. We first present the linear analysis whose object is the determination of the eigenfrequency pattern.

The evolution of the velocity field of the oscillations and of the perturbed pressure, density and gravity terms associated to the oscillations is governed by the following equations (the analytical expressions and the complete resolution are given in Unno et al. 1979):

- the equation of the movement, which exhibits three restoring forces: pressure perturbation, buoyancy, and perturbation of the gravitational potential. The Cowling approximation considers that the last term is negligible. The pressure term dominates for sound waves.

- the equation of continuity, which expresses the mass conservation.

- the Poisson equation (in fact useless when neglecting the perturbation of the gravitational potential).

- the equation governing the evolution of the perturbation. Since the perturbation evolves much more rapidly than the characteristic evolution time of the unperturbed state, the waves propagate adiabatically.

The most simple solution is the ray tracing theory, which only considers the propagation of the wave vector. It is sustained by the assumption that the propagative wave is a pure plane wave. Mosser et al. (1988) use the the helioseismologic analysis proposed by Gough (1986) with this method, but include the structure discontinuity of Jupiter. The theory is valid for the determination of Eqt. 22.1, but inadequate for a further description of the eigenfrequencies pattern. In the following, we will focus on the asymptotic method, which proved to be powerful for the understanding of the planetary oscillations (Provost et al. 1993), as well as on the numerical calculations developped for precisely taking into account all the features of the Jovian interior structure, namely the core, the PPT and the rapid rotation.

\subsubsection{Asymptotic approach}

An asymptotic method for calculating the Jovian oscillation spectrum has been proposed by Provost et al. (1993). It follows the asymptotic development of Tassoul (1980), but includes the discontinuity of the Jovian core. The exact eigensolutions describing the movements are asymptotically developped in Bessel's functions to the second order in frequency, near the surface (where the evolution is dominated by the adiabatic index $n_{e}$ of the upper atmosphere) and near the center (where the evolution is dominated 
by the degree $\ell$ of the mode), and finally connected by the discontinuity. The main advantage of this analytical procedure is to obtain seismological parameters which can be related to the internal structure parameters. On the other hand, the method does not permit a precise description of more than one discontinuity.

\subsubsection{The modulation due to the core}

Asymptotic eigenfrequencies accounting for the discontinuity in the sound speed profile are given by:

$$
\begin{aligned}
\nu_{n, \ell}=\left[n^{\prime}\right. & +\frac{L^{2} V_{1}+V_{2}}{4 \pi^{2} \nu_{n, \ell}} \\
& \left.-\frac{\varepsilon}{\pi} \sin \alpha_{n, \ell}-\frac{\varepsilon^{2}}{\pi} \frac{N-2}{2 N} \sin 2 \alpha_{n, \ell}\right] \nu_{0}
\end{aligned}
$$

with $L^{2}=\ell(\ell+1)$ and

$$
\alpha_{n, \ell}=2 \pi\left[\frac{n^{\prime}}{N}-\frac{\ell}{2}-\frac{L^{2} V_{3}+V_{4}}{4 \pi^{2} \nu_{n, \ell}}\right] ; n^{\prime}=n+\frac{\ell}{2}+\frac{n_{e}}{2}+\frac{5}{4}
$$

The first part of Eqt. 22.5 reproduces the solar asymptotic expression, while the second expresses the influence of the core.

- The characteristic frequency $\nu_{0}$ measures the travel time of the sound along a planetary diameter:

$$
\nu_{0}=\left[2 \int_{0}^{R} \frac{\mathrm{d} r}{c}\right]^{-1}
$$

$R$ is the planetary radius, commonly defined at the 1-bar pressure level. As shown by Mosser (1990), the upper level which should be considered when integrating $\nu_{0}$ is the tropopause. Eqt. 22.8 recapitulates the different steps for the exact calculation of $\nu_{0}$, taking into account the tropospheric contribution and also the effect of the oblate planetary shape:

$$
\begin{array}{ccc}
\text { Interior } & \text { Troposphere } & \begin{array}{c}
\text { Rotation } \\
\downarrow
\end{array} \\
\nu_{0}=\left[2\left(\int_{0}^{\downarrow} \frac{\mathrm{d} r}{c}+\int_{R}^{\text {tropopause }} \frac{\mathrm{d} r}{c}\right)\right]^{-1} \times\left[1-\frac{e}{6}\right]
\end{array}
$$

The cavity where the modes propagate ends at the tropopause (a level which is not included in current interior models). The contribution of the planetary oblateness $e$ is due to the fact that the planetary seismological mean radius $R(1-e / 3)$ differs from the geometrical mean radius $R(1-$ $2 e / 9$ ). The modes favor in fact the equatorial regions (Mosser 1992). 
- The second order characteristic frequency is also a simple function of the sound speed, accounting for all the variations of $c$ :

$$
V_{1}=\int_{0}^{R} \frac{\mathrm{d} c}{r}
$$

whereas the other $V_{i}$ are complex integrals of interior parameters.

- The core modulation has a period $N$ and a relative amplitude $\varepsilon$. The integer $N$ measures the acoustic radius of the core compared to the whole planetary acoustic radius:

$$
N=\int_{\text {planet }} \frac{\mathrm{d} r}{c} / \int_{\text {core }} \frac{\mathrm{d} r}{c}
$$

- $\varepsilon$ measures the discontinuity at the core frontier (Table 22.4).

Finally, the pertinence of the seismological parameters shown by the previous equations must be noted (Provost et al. 1993). Their determination, extrapolated from a non-asymptotic but numerical oscillation pattern, agrees with their theoretical values.

\subsubsection{The rotation}

The comparison of Jupiter and the Sun shows the importance of rotation for Jovian seismology. Both objects present similar freefall frequencies, but their rotational frequencies are respectively 28.2 and $0.4 \mu \mathrm{Hz}$. The removal of degeneracy due to the Jovian rotation cannot be neglected. Mosser (1990) describes the four different effects of rotation: the non-galilean planetary referential, the oblate shape of the planet, the Coriolis and centrifugal forces. The two last terms become negligible for high overtones $\left(\nu_{n, \ell} \gg \nu_{0}\right)$. But the influence of rotation is severe (Fig. 22.6). The non-degenerated eigenfrequencies $\nu_{n, \ell, m}$ express as a function of the degenerated frequencies $\nu_{n, \ell}$ (Mosser 1990):

$$
\nu_{n, \ell, m}=\nu_{n, \ell}\left[1+E\left(m^{2}\right)\right]-m \nu_{\text {rot }}
$$

where the azimuthal order $m$ varies from $-\ell$ to $\ell$ ( $m$ is the second index of the spherical harmonics $Y_{\ell}^{m}$ associated with the mode). $E\left(m^{2}\right)$ is a function related to the oblateness and with the same order of magnitude.

\subsubsection{Oscillation spectra of the giant planets}

The asymptotic data which describe the modulation due to the core are given in Table 22.5. What differs principally between Jupiter and Saturn on one side and Uranus and Neptune on the other side is the period $N$. Jupiter and Saturn have a small rock core, which implies a large $N$, whereas Uranus 

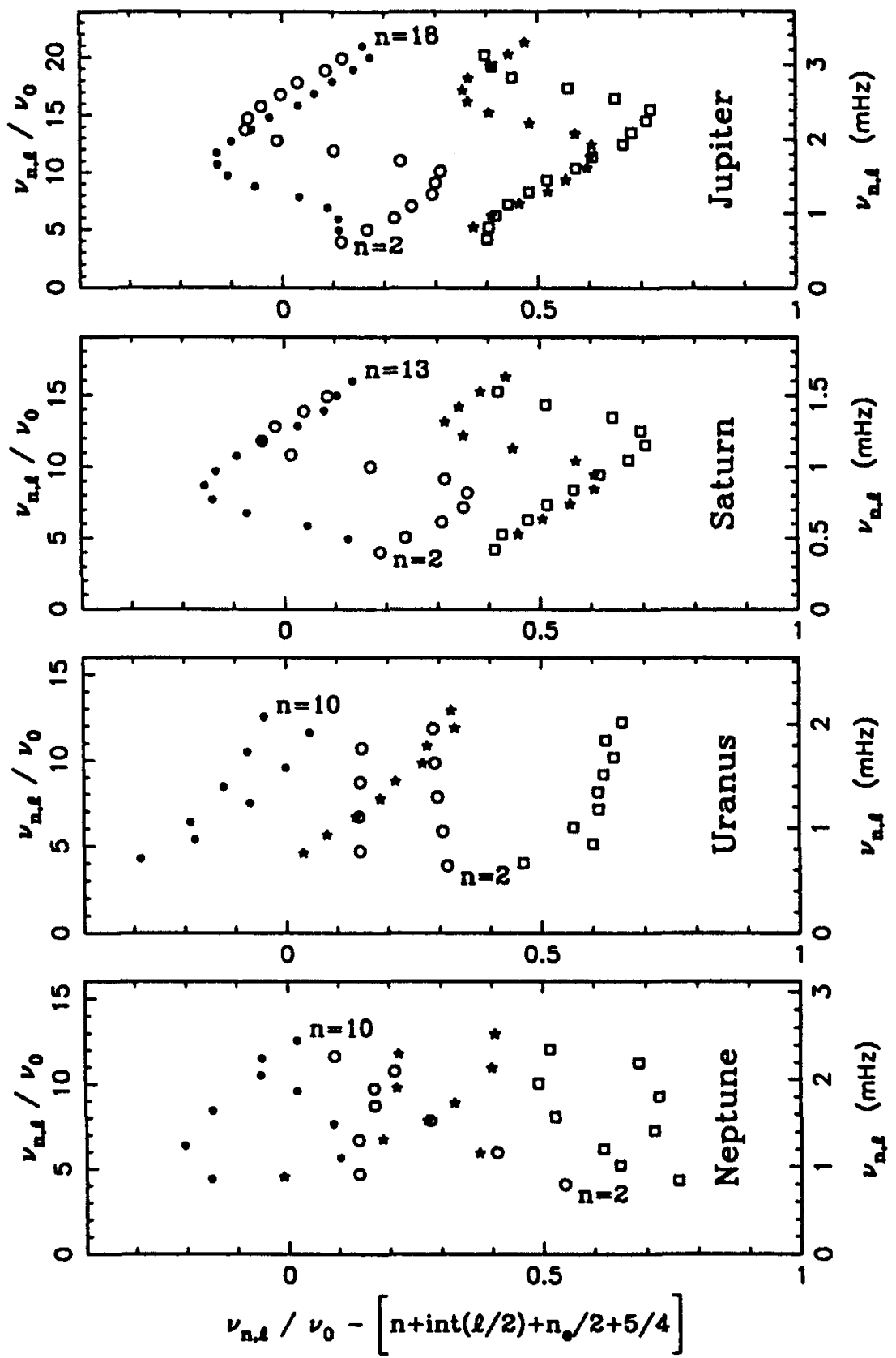

Fig. 22.5 Asymptotic échelle diagrams for giant planets p-modes with degree $\ell=0 \rightarrow 3: \nu / \nu_{0}$ is plotted as a function of the reduced frequency $\delta \nu=\nu / \nu_{0}-\left[n+\operatorname{int}(\ell / 2)+n_{e} / 2+1 / 4\right]$. The modulation is function of the frequency, namely of the radial order $n$ : its period is about $N$. The amplitude of the modulation is related to the coefficient $\varepsilon$. For each planet, the radial order $n$ varies from 2 to the maximal order of the trapped modes (cf. Table 22.5). ( $\ell=0: 0 ; \ell=1: \square ; \ell=2: \bullet ; \ell=3: \star)$ 
Table 22.4. Jovian sound speed discontinuities

\begin{tabular}{rcc}
\hline Discontinuity & $\varepsilon$ & $\Delta c / r\left(\right.$ mrad. $\left.^{-1}\right)$ \\
\hline Core & $0.13 \rightarrow 0.44$ & $3 \rightarrow 5$ \\
PPT & $0 \rightarrow 0.05$ & $-0.1 \rightarrow 0.1$ \\
\hline
\end{tabular}

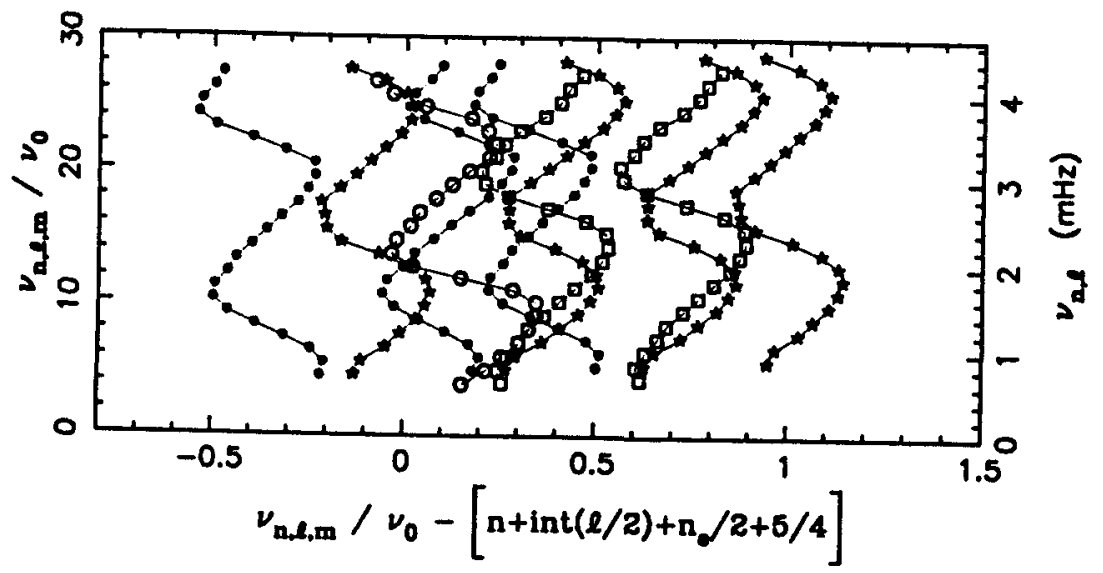

Fig. 22.6 Jovian échelle diagram including rotation, according to the perturbation theory (Mosser 1990). Only modes with $(\ell+m)$ even are shown. $(\ell=0: 0 ; \ell=1:$ 口 $\ell=2: \bullet ; \ell=3: \star)$

and Neptune have a large ice shell and $N \simeq 2$. Table 22.6 summarizes the various frequencies governing the qualitative aspect of the planetary oscillation spectrum.

- $\nu_{\bar{p}}=\sqrt{G M / R^{3}} / 2 \pi$ measures the mean density of the planet and varies as $\nu_{0}$. The ratios $\nu_{\bar{\phi}} / \nu_{0}$ are similar for the four giant planets.

- $\nu_{\text {rot }}$ : the rotation frequency, compared to $\nu_{0}$, measures the complexity of the spectrum due to the rotation. For Saturn, $\ell=2$ modes already overlap $\left(\nu_{n, 2,2} \simeq \nu_{n-1,2,-2}\right)$; this overlap occurs for $\ell=3$ Jovian modes and $\ell=5$ for Uranus et Neptune, as indicated by the ratio $\nu_{0} / 2 \nu_{\text {rot }}$.

- $\nu_{c}$ : the cutoff frequency at the tropopause represents the highest possible eigenfrequency. It is related mainly to the temperature. The ratio $\nu_{c} / \nu_{0}$ gives an estimate of the number of modes effectively trapped; modes with $n$ greater than this ratio cannot be reflected at the tropopause level.

Finally, the échelle diagrams of all four giant planets are presented on Fig. 22.5. The échelle diagram representation exhibits the meaningful dif ference between the eigenfrequencies developped to the second order in frequency and Eqt. 22.1. The method to build the échelle diagram is explained 
Table 22.5. Asymptotic seismological data

\begin{tabular}{rrrrrrrrr}
\hline Planet & $\begin{array}{r}\nu_{0} \\
(\mu \mathrm{Hz})\end{array}$ & $\mathrm{N}$ & $\varepsilon$ & $V_{1}^{\prime}$ & $V_{2}^{\prime}$ & $V_{3}^{\prime}$ & $V_{4}^{\prime}$ \\
& & \multicolumn{2}{c}{$\ldots$} & $V_{i}^{\prime}=V_{i} / 4 \pi^{2} \nu_{0}$ & $\ldots$. \\
\hline Jupiter & 155 & 12.6 & 0.40 & 0.1 & 2.3 & -0.8 & -1.3 \\
Saturn & 111 & 10.0 & 0.41 & -0.3 & 1.5 & -0.7 & -0.3 \\
Uranus & 164 & 2.0 & 0.21 & -0.4 & 0.1 & -0.1 & 0.6 \\
Neptune & 195 & 2.2 & 0.13 & -0.4 & 1.9 & -0.1 & -3.9 \\
\hline
\end{tabular}

Table 22.6. Characteristic frequencies

\begin{tabular}{ccccccccc}
\hline Planet & \multicolumn{2}{c}{$\nu_{\bar{\phi}}$} & $\nu_{0}$ & $\nu_{\text {rot }}$ & $\nu_{c}$ & $\nu_{0} / \nu_{\bar{\phi}}$ & $\nu_{0} / 2 \nu_{\text {rot }}$ & $\nu_{c} / \nu_{0}$ \\
& $\ldots$ & $(\mu \mathrm{Hz}) \ldots$ & $(\mathrm{mHz})$ & & & \\
\hline Jupiter & 99 & 155 & 28 & 3.0 & 1.59 & 2.8 & 18 \\
Saturn & 70 & 111 & 26 & 1.6 & 1.59 & 2.1 & 13 \\
Uranus & 94 & 165 & 16 & 1.8 & 1.74 & 5.1 & 10 \\
Neptune & 105 & 190 & 18 & 2.2 & 1.80 & 5.3 & 10 \\
\hline the Sun & 99 & 136 & 0.4 & 7.0 & 1.37 & 165 & 50 \\
\hline
\end{tabular}

Table 22.7. Principle of the échelle diagram

\begin{tabular}{|c|c|c|c|c|}
\hline frequencies & eigenfrequency $\nu$ & & quidista & $\Delta \nu \sim \nu_{0}$ \\
\hline $\begin{array}{l}\text { asymptotic } \\
\text { relation }\end{array}$ & $\downarrow$ & $=p$ & $\Delta \nu$ & $\delta \nu$ \\
\hline échelle diagram & $\begin{array}{l}\downarrow \\
\text { vertical axis }\end{array}$ & & $p \in \mathbb{N}$ & $\stackrel{1}{\text { horizontal axis }}$ \\
\hline
\end{tabular}

in Table 22.7†. The échelle diagram of Fig. 22.6 includes the rotational removal of degeneracy.

\subsubsection{Numerical approach}

Different numerical codes have been developped for the calculations of Jovian pressure modes ( $p$-modes), fundamental modes ( $f$-modes) or surface modes (these one have significant amplitudes in the vicinity of the discontinuities). Numerical calculations (Fig. 22.7, 22.8, 22.9 and 22.10) can explore the whole $[n, \ell]$ domain, contrary to asymptotic calculations. The precision is limited by the absence of the tropospheric contribution, except for the very low frequency modes $(\leq 1 \mathrm{mHz})$ which are trapped in the deep troposphere.

† The échelle diagram exhibits the small frequency term $\delta \nu$, which carries the essential information of the eigenfrequency $\nu=p \nu_{0}+\delta \nu$ ( $p$ is an integer). 


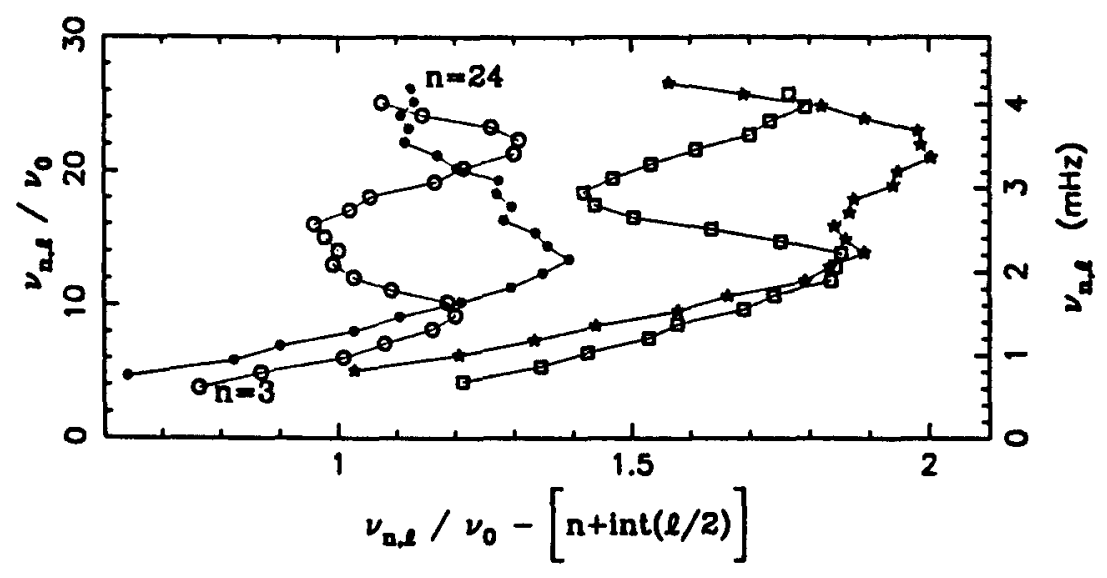

Fig. 22.7 Jovian numerical échelle diagram: same as Fig. 22.5, but using numerically computed eigenfrequencies (Provost et al. 1993). $\quad(\ell=0: 0$; $\ell=1:$ 口; $\ell=2: \bullet ; \ell=3: \star)$

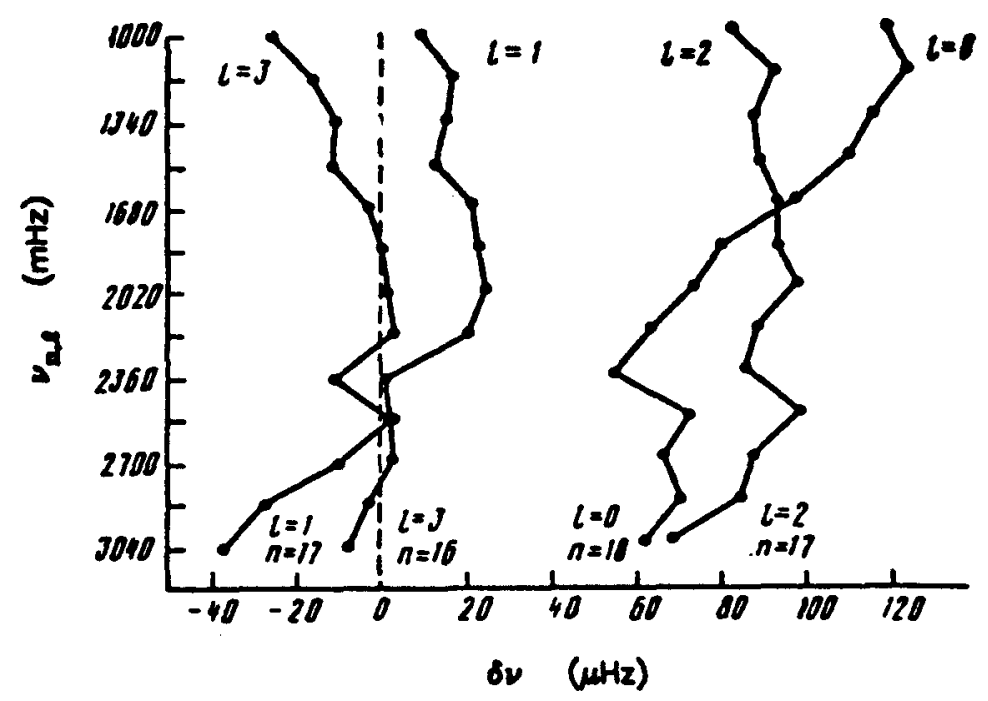

Fig. 22.8 Jovian numerical échelle diagram according to Vorontsov (from Vorontsov et al. 1989, Fig. 5). This diagram represents the same low degree modes as the one of Fig. 22.7. Differences between the two figures are mainly related to differences between the interior models.

\subsubsection{Normal mode theory}

Most of the approaches (Vorontsov et al. 1989, Marley 1991, Provost et al. 1993) are derived from helioseismology, following the normal mode theory, but including density and sound speed discontinuities. Rotation (and differential rotation) are considered as perturbations (Vorontsov 1981, 1984a and b, Vorontsov \& Zharkov 1981). On the other hand, Lee (1993) introduces the rotation at the zeroth order of the calculations. Because of rotation, 


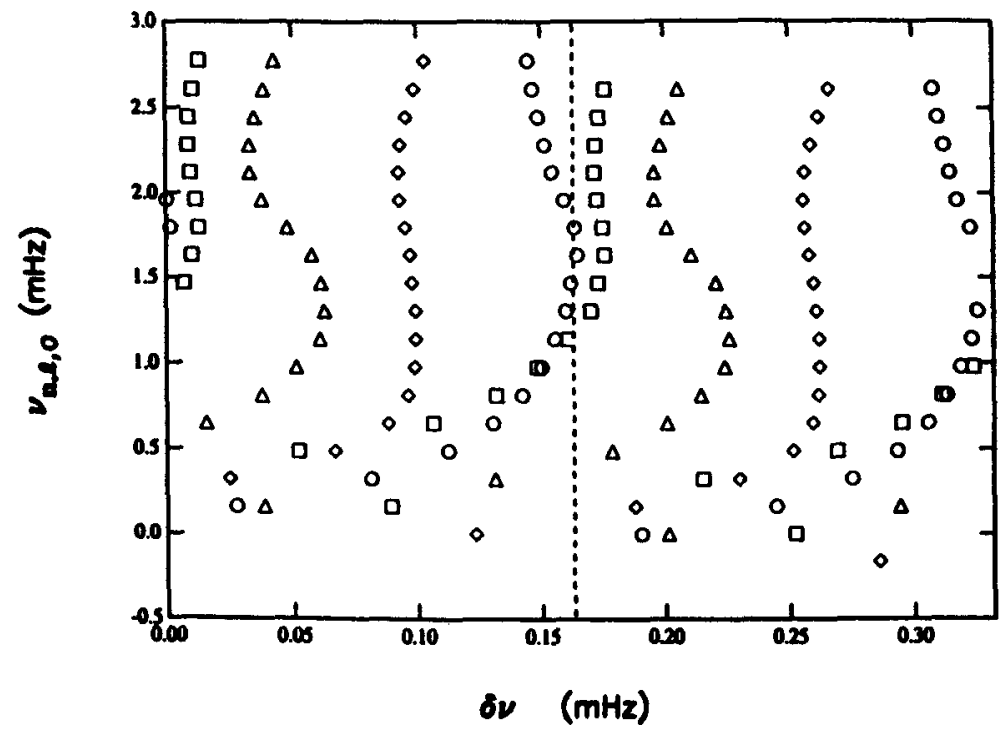

Fig. 22.9 Jovian numerical échelle diagram calculated by Lee (from Lee 1993, Fig. 4). Frequencies are given in the Jovian corotating frame, for $m=0$, even low degree $p$-modes. The couplage between the different degrees precludes the intersection of the modes, contrary to the perturbation approach. $(\ell=0: 0 ; \ell=2: \diamond ; \ell=4: \Delta ; \ell=6: \square)$

the modes are no longer described by an only spherical harmonics, but by a series of such terms. The mode described by the numbers $n, \ell$ and $m$ is developped in terms of the spherical harmonics $Y_{\ell}^{m}, Y_{\ell \pm 2}^{m}, Y_{\ell \pm 4}^{m} \ldots$ (the index $m$ remains the same because of axisymmetry). The main result of the couplage between the modes is that, contrary to perturbation results, crossings between modes of different degrees are avoided (Fig. 22.9). However, the principal property of the rotational splitting remains true:

$$
\nu_{n, \ell,-m}-\nu_{n, \ell, m}=2 m \nu_{\text {rot }}
$$

\subsubsection{The Saturnian ring system as a seismometer}

Marley (1991) focusses his interest on low frequency Saturnian f-modes. These low frequency oscillations perturb the gravitational potential of the planet and can open gaps in the rings. Therefore, the Saturnian ring system acts as a seismometer. The great advantage of ring seismometry consists in the possibility of detecting very low frequencies $f$-modes, which are not detectable with a ground based detector. However, such a detection can only be applied to Saturn! An important observational result reported by Marley is the fact that low frequency Saturnian modes do not open large unexplained gaps in the ring system, which implies a surface amplitude lower than about 1 meter for very low frequency modes (a few tens of $\mu \mathrm{Hz}$ ). 


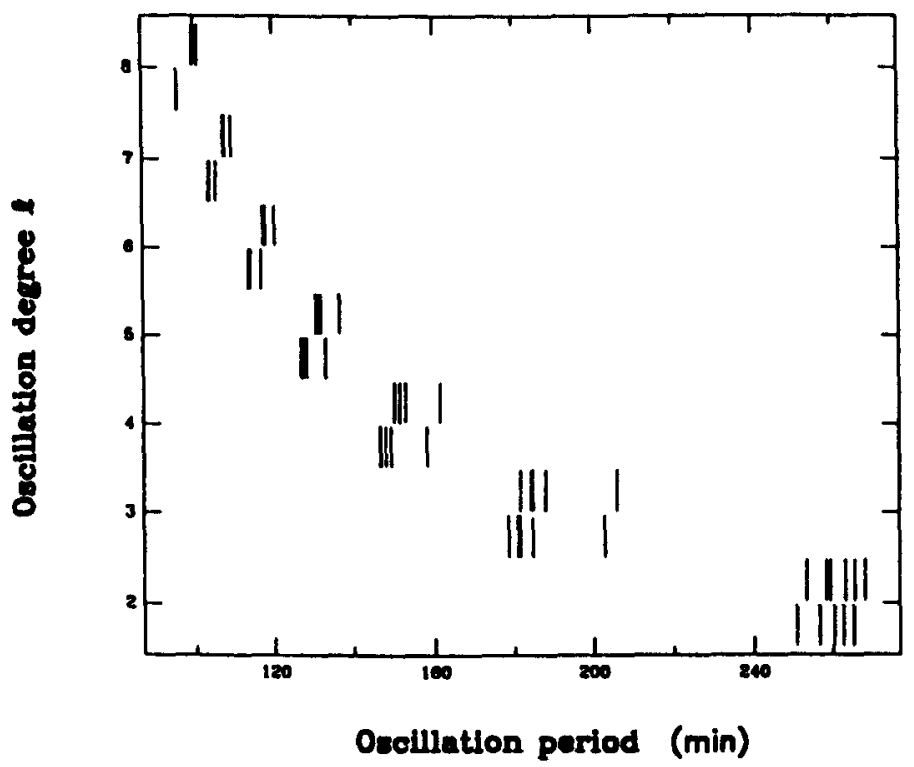

Fig. 22.10 Saturnian low frequency $\ell=m$ f-modes (from Marley 1991, Fig. 10). f-modes are modes without radial nodes. For each degree $\ell$, the different dashes correspond to different Saturnian interior models. As for Jupiter, the differences between interior models express by huge differences in the eigenfrequency pattern.

\subsubsection{Observations}

\subsubsection{Three observations}

Three positive observation runs of Jovian oscillations have been conducted in 1987, 1990 and 1991, using two different seismometric techniques. The principle of the detection is as follows. The oscillations induce in the upper troposphere a vertical velocity field (Fig. 22.11). The spectral lines, reflected or formed at these levels, are Doppler shifted. The Doppler shift of the solar sodium line reflected by the planet has been observed with a sodium resonance cell (Schmider et al. 1991), and the Fourier transform spectrometry method has analysed the Doppler shift in the interferogram of the Jovian methane lines at $1.1 \mu \mathrm{m}$ (Mosser et al. 1993). The Doppler signal is recorded over several consecutive nights. The resulting temporal series is cleaned and its Fourier transform is calculated in order to search for the planetary eigenfrequencies.

\subsubsection{Analysis}

The most obvious signature which appears in the Fourier spectra is the non continuity of the observations. Observations conducted in a single 

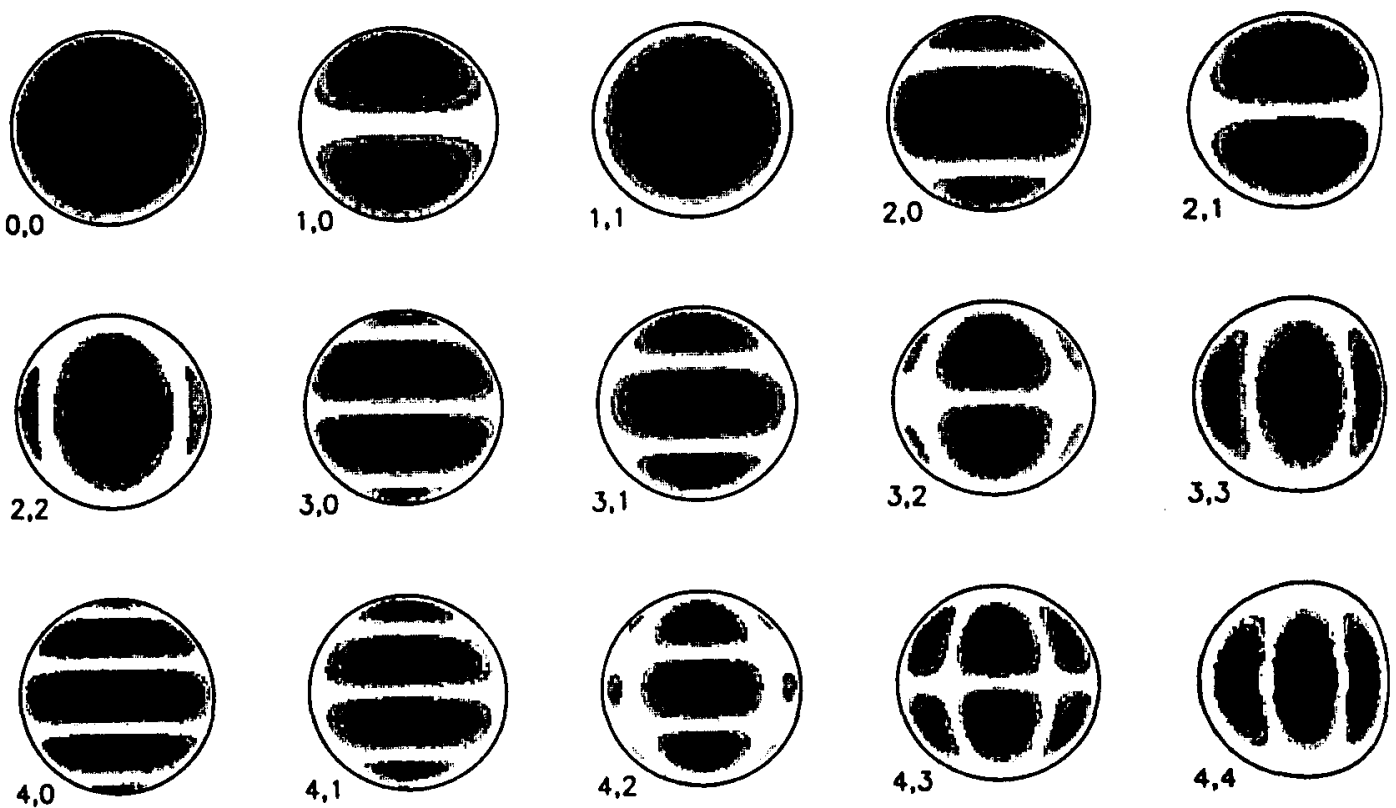

Fig. 22.11 Oscillation velocity field in the upper troposphere, corresponding to the projection of the spherical harmonics $Y_{\ell}^{m}$ on the view axis.

site are limited to about 8 hours. The resulting effect (Fig. 22.12) on the oscillation pattern is desastrous, as seen on a theoretical oscillation spectrum (Fig. 22.13). The very high mode density precludes any treatment of the window effect; the solution which consists of separately considering the nights to obtain a continuous series is also inoperative, since the resolution after one single night is not sufficient to resolve the modes.

\subsubsection{Detection of the oscillations}

A commonly used criterion for the detection of oscillations (Gelly et al. 1986, Pottasch et al. 1992) is the building of an échelle diagram which should show regular patterns aligned vertically. This criterion cannot be extensively used for Jovian modes, since the modulation excludes vertical alignments in an entire échelle diagram (Provost et al. 1993). In fact, the signature of the oscillations has been given by the signature of the rotation. The rotation affects the signal in two ways first, it modulates the photometric signal, when some atmospheric features pass through the field of view; secondly, it removes the degeneracy of the modes, with the relation indicated by Eqt. 22.11. The first effect cannot be responsible for the signal at frequencies much higher than the rotational frequency (Schmider et al. 1991). The second rotational signature is a consequence of Eqt. 22.12. The signature of the rotational removal of degeneracy is represented in the 

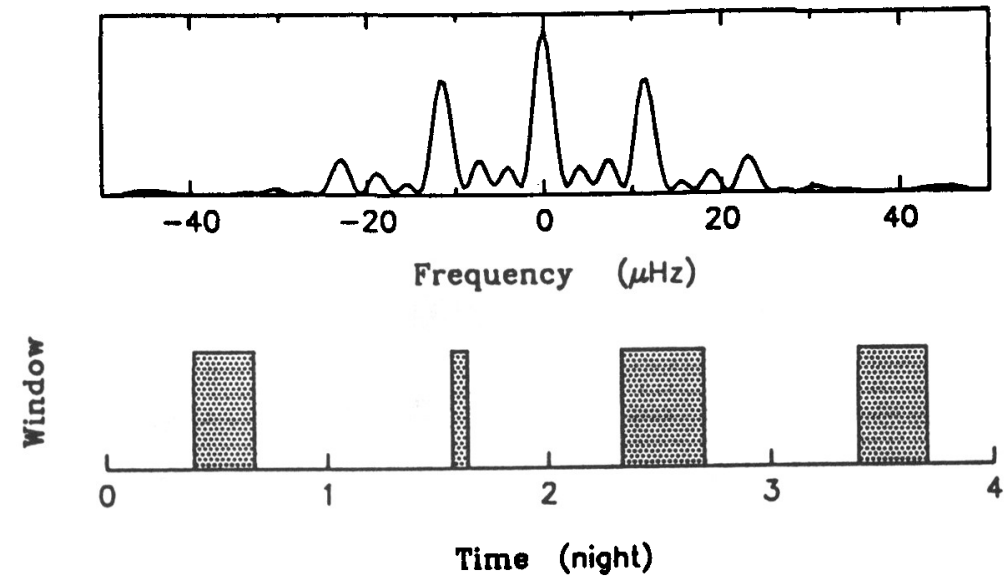

Fig. 22.12 Fourier spectrum of the window function of the observation made at the CFHT in 1990 with the Fourier transform spectrometer (below: the shaded zone corresponds to effective observation). The diurnal signature appears in the spectrum as aliases at $\pm 11.6 \mu \mathrm{Hz}$. The duration of the observation determines the sharpness of the peaks in the Fourier spectrum. The extension of the spectrum is given by the duty cycle: less than 8 hours daily observation imply the dilution of one peak of the spectrum over about $50 \mu \mathrm{Hz}$, with only $1 / 4$ of the power in the central peak.

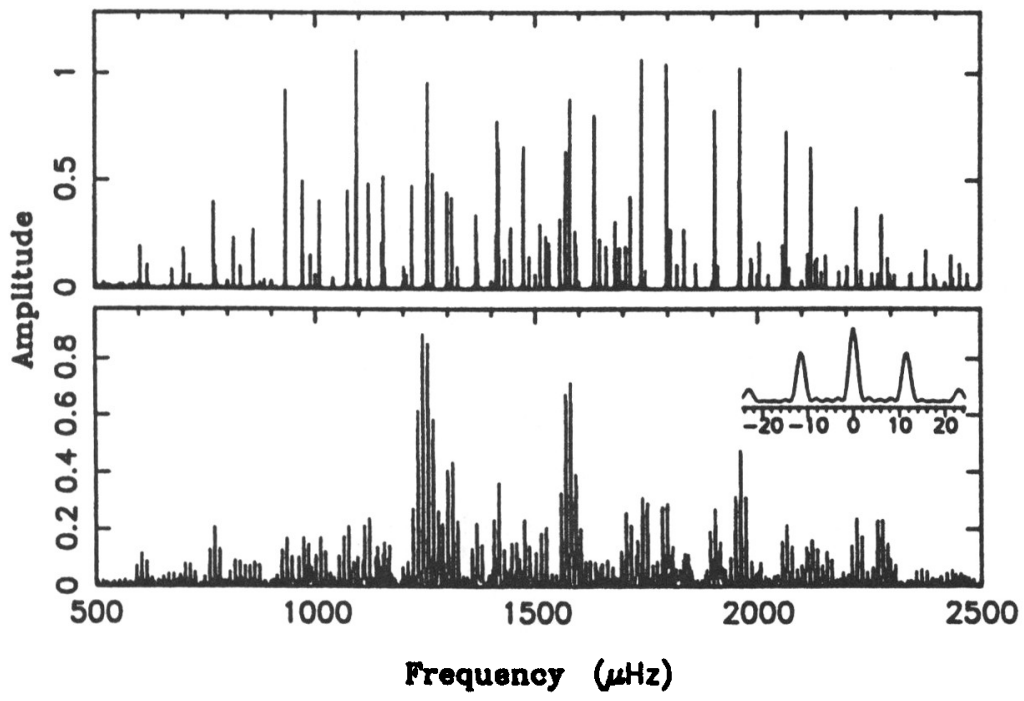

Fig. 22.13 Theoretical spectra, as continuously observed, or with a window of 8 hours observation per night. Amplitudes have been arbitrarily chosen: Gaussian envelopes; cutoff above the cutoff frequency at the tropopause; decreasing amplitude with increasing $\ell$ value; same amplitude for multiplets $\nu_{n, \ell, m}=-\ell \rightarrow \ell$.

Fourier spectrum of the observed Fourier spectrum by half of the rotation period of Jupiter, and by overtones (Fig. 22.14). Finally, this second effect 


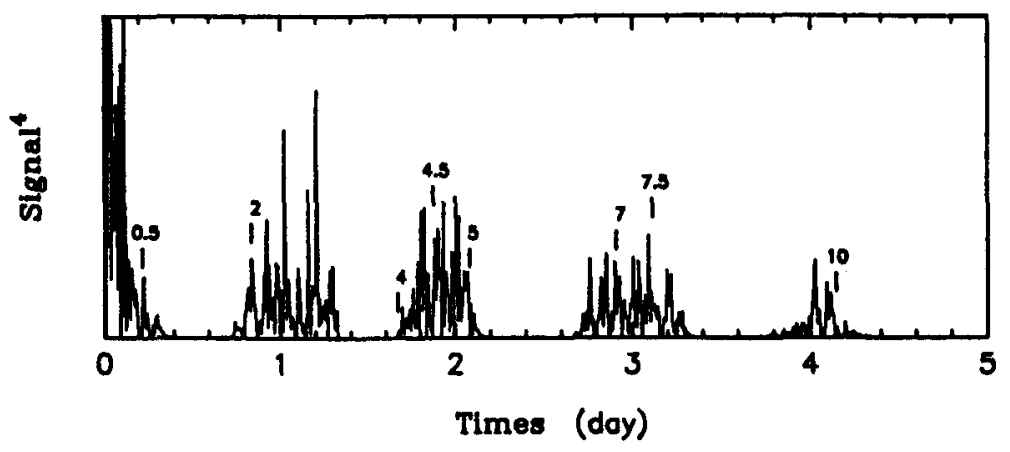

Fig. 22.14 Fourier spectrum of the Fourier spectrum observed in 1991 at the CFH telescope. The signature of the removal of degeneracy due to rotation appears at periods multiple of half the Jovian period of rotation. The empty zones correspond to the absence of observations during daytime.

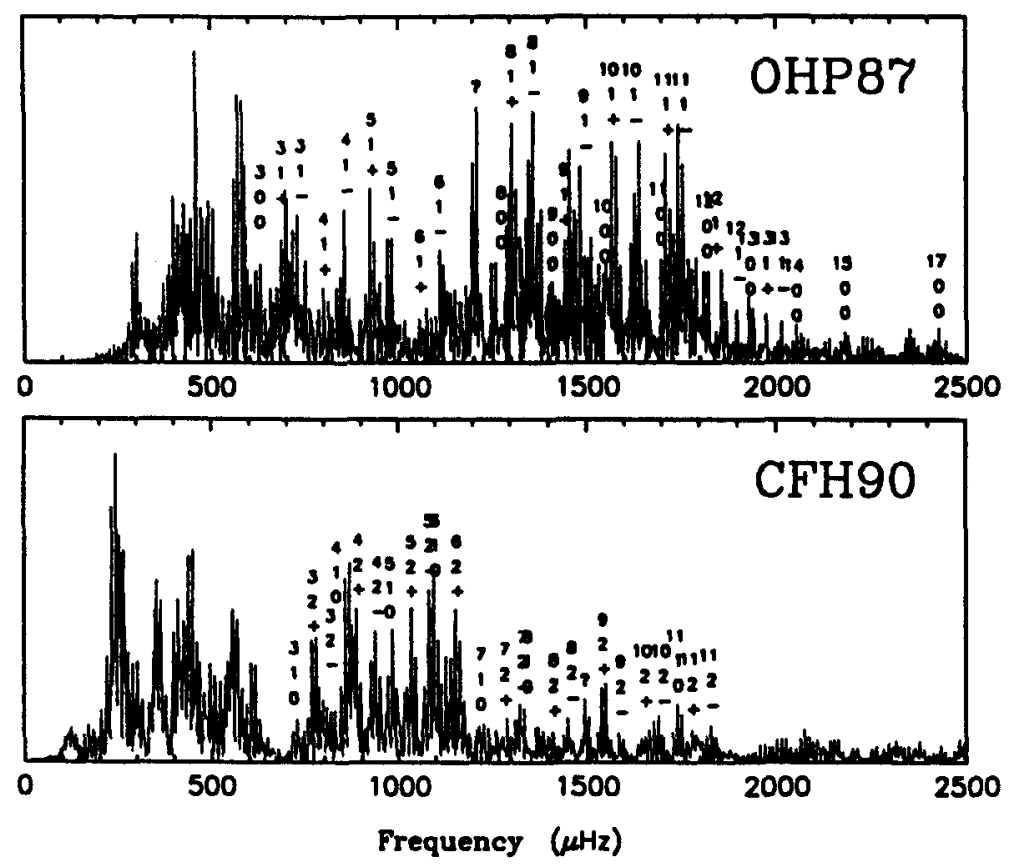

Fig. 22.15 Power spectra obtained in 1987 at the OHP, with the sodium cell resonance technique (Schmider et al. 1991), and at the CFHT in 1990, with the Fourier Transform Spectrometer (Mosser et al. 1993). A possible identification of the modes $[n, l, m]$ is proposed ( \pm is for $m= \pm 1$ ).

has proved the detection of a propagative signal. Because the other possible propagative signals - tropospheric or stratospheric waves - have very low frequencies $(\ll 1 \mathrm{mHz})$, the signal has been identified with Jovian global oscillations. 


\subsubsection{Observational results}

The Jovian oscillation spectra are presented on Fig. 22.15. As reported in Mosser et al. (1991, 1993) the unambiguous identification of all individual eigenfrequencies is not possible. However, a plausible identification may be proposed, that agrees for all three spectra, and is based on the identification of the signature of the rotational removal of degeneracy, namely the $[\ell=1$ or $2, m= \pm 1$ ] doublets.

\subsubsection{Discrepancies}

In fact, what is more interesting is the extrapolation from the three oscillations patterns of some characteristic asymptotic parameters. We will focus on the characteristic frequency $\nu_{0}$, revealed by a possible equidistance frequency $\Delta \nu$. The theoretical value of $\nu_{0}$ calculated from current interior models lies, according to Eqt. 22.8 , between 155 and $160 \mu \mathrm{Hz}$. The observed value of the equidistance $\Delta \nu$ is about $136 \mu \mathrm{Hz}$. It can be related to a characteristic frequency $\nu_{0}$ in the frequency range $[\Delta \nu(1-2 \varepsilon / N), \Delta \nu(1+2 \varepsilon / N)]$. Typically, $\Delta \nu$ and $\nu_{0}$ may differ from about $10 \mu \mathrm{Hz}$. At least, the discrepancy between the theoretical and observed values of $\nu_{0}$ is about $10 \mu \mathrm{Hz}$. This discrepancy should have a manifold explanation. It can be related to problems encountered in physics (the determination of the EOS), or in seismology (the calculation of the sound speed and of $\nu_{0}$ ), or in planetology (the validity of the hypothesis of the standard model: adiabaticity and homogeneous composition).

- EOS: The relationship between the sound speed $c$ and the EOS has already been emphasized. The precision on $c$ largely depends on the one of the EOS (1\% inaccuracy on the EOS leads to a much bigger inaccuracy on c). If accuracy is now insured for the EOS of molecular and metallic hydrogen, that is not the case for helium and other heavier elements.

- Calculation of the sound speed: The calculation of $c$ supposes the structure to be adiabatic in the whole planet, what is only an approximation. The influence of the superadiabaticity needed for evacuating the internal flux is limited, but still exists. Furthermore, the continuity of the adiabat from the 1-bar level to the deep interior supposes no compositional gradient and convection everywhere. Any change in the value of the adiabat would substantially modify the temperature profile at all the deeper levels, and then modify significantly the sound speed profile.

- Composition gradient: A composition gradient must exist somewhere in the planet to reconcile the observed abundances in the outer envelope with the values assumed to occur in the metallic region on the basis of cosmogonical arguments. Such a gradient modifies inevitably the adiabat. 
- Radiative gradient: As calculated in Guillot et al. (1993), a radiative window should exist in Jupiter near the $3000 \mathrm{~K}$ level. This window would change the entire planetary temperature profile, conducting to lower inner temperature, and maybe to lower sound speeds.

- Inhomogeneities: Let us consider in Jupiter a layer $\mathrm{d} r$, composed of hydrogen and heavier elements, with volumic fractions respectively $(1-\alpha)$ et $\alpha$. The acoustic radius of the layer $d \tau$ measures the inverse of the mean sound speed $\bar{c}(\mathrm{~d} \tau=\mathrm{d} r / \bar{c})$ and depends on the constitution:

$$
\begin{cases}\text { Homogeneous mixing: } & \mathrm{d} \tau_{1} \simeq \frac{\mathrm{d} r}{c_{\mathrm{H}}}\left[1+\alpha\left(1-\frac{c_{\mathrm{Z}}}{c_{\mathrm{H}}}\right)\right] \\ 2 \text { distinct levels: } & \mathrm{d} \tau_{2} \simeq \frac{\mathrm{d} r}{c_{\mathrm{H}}}\left[1+\alpha\left(\frac{c_{\mathrm{H}}}{c_{\mathrm{Z}}}-1\right)\right]\end{cases}
$$

Since the sound speed in hydrogen, $c_{\mathrm{H}}$, is much higher than the one in the heavier elements, $c_{Z}$, the acoustic radius in case of heterogeneity is larger, which implies a lower characteristic frequency.

- Perturbation of the adiabat by clouds: The planetary adiabat is for many elements over the critical point, implying no phase separation. However, the presence of water clouds introduces a discontinuity in the adiabat, and leads to lower interior temperatures, and subsequently to smaller sound speed.

Finally, the hypotheses supporting the standard model should be revised.

\subsubsection{Excitation mechanism}

The velocity of the observed low degree modes has been estimated about a few $\mathrm{m} . \mathrm{s}^{-1}$ at the 0.5 -bar pressure level. The dependence of the velocity of the wave with altitude is very important (Mosser et al. 1992). A velocity of about $1 \mathrm{~m} . \mathrm{s}^{-1}$ at the 0.5 -bar level corresponds to only a few mm.s ${ }^{-1}$ at the PPT level. Depending on the frequency $\nu$ of the mode compared with the cutoff frequency, this velocity $v$ corresponds to the amplitude $a$ of an isobar level (Mosser, in preparation):

$$
a=\rho_{0} c v \text {, or } a=v / 2 \pi \nu
$$

That leads to an amplitude of the 1-bar level of about $100 \mathrm{~m}$ for $v=1 \mathrm{~m} . \mathrm{s}^{-1}$.

The excitation mechanism proposed by Goldreich et al. (1988) for the solar oscillations gives an order of magnitude of the expected Jovian velocity, which is too low by about a factor 100 or 1000 when considering the convection in the upper Jovian atmosphere (parallely to what is done for the Sun). Then, it seems inadequate for Jupiter, and no other mechanism is able to quantitatively explain the high amplitudes detected. On the other hand, Mosser (1991) has shown that: 
- The estimated energy in the oscillations is compatible with the flux radiated by the planetf.

- The PPT is a possible region of excitation of the modes. Since the entropy shift per proton at the PPT is very high (about $k_{\mathrm{B}} / 2$ per proton), the PPT, if it is a first order transition, acts as an impermeable surface. Surface waves can then develop. They have exponentially decreasing amplitudes and are therefore not detectable, but can be coupled with sound waves.

- The couplage is efficient, because it occurs between phenomena with similar periods, and the excitation too, because it appears in the deep planet, contrary to convection, which is too slow in the deep region or inefficient in the upper atmosphere.

\subsection{Discussion}

\subsubsection{The seismological test}

Helioseismology was an unknown solar field 30 years ago, but has now provided measurements which are among the most precise in astrophysics, and have permitted to reconstruct the solar interior profile (ChristensenDalsgaard et al. 1985). Can we expect the same improvements for the giant planets? Bearing in mind that the precise measurement of many eigenfrequencies will not be obtained rapidly, we focus the discussion on global asymptotic criteria. Pure hydrogen adiabats, as well as density and sound speed profiles resulting from the different EOS have already been compared, showing how the seismological parameter $c$ is sensitive to the EOS. Measuring the sound speed profile would require what we have exluded, the measurement of numerous eigenfrequencies. However, as shown by Eqt. 22.7 and Eqt. 22.9, most of the asymptotic parameters are related to the sound speed and may be translated into interior structure parameters.

\subsubsection{The signature of the PPT \\ 22.4.2.1 Low degree modes}

In current models, the influence of the PPT is hidden by that of the core. This is not only due to the fact that the core discontinuity is much stronger, this is also conceptually related to the way the models are made. Any

† This estimation is problematic, since it needs the determination of a characteristic damping time of the oscillations! We can guess that this time is not short according to the following informations: the viscosity of molecular hydrogen is very low; there is no radiative zone in Jupiter, and therefore no radiative viscosity; the quality factor of the planet is very high. 
change of the EOS (for example, with or without PPT) leads to a change of the size of the core, which implies a huge change in the seismological modulation. This appears very clearly on Fig. 3 of Provost et al. (these proceedings), where eigenfrequencies of different models based on different EOS are compared. The EOS modification affects principally the low degree modes modulation. The second effect is the change of the temperature profile, corresponding then to a small change of the characteristic frequency $\nu_{0}$, which causes the small mean slope in the diagram, visible for all degree modes. But this can be considered only as an indirect consequence of the PPT.

In fact the frontier at the PPT induces qualitatively the same kind of modulation as the one due to the core, but with a much lower amplitude (Table 22.4). This modulation already appears on the numerical échelle diagram (Fig. 22.7). Due to the expected location of the transition, the period $N_{\text {PPT }}$ is about 2 . The amplitude is very small, so that finally the direct signature of the PPT corresponds to a slight and rapid modulation. The detection of this modulation requires a very high frequency resolution.

\subsubsection{High degree modes}

High degree modes, which propagate in the upper envelope, are less sensitive to the core. We have compared different models based on different equations of state in order to test the capability of high degree modes to sound the PPT. Results are presented on Fig. 22.16, an histogram of the number of modes refracted at a given level. The direct signature of the PPT appears with evidence on modes with degrees higher than 8 . The number of modes refracted at the PPT strongly depends on the sound speed discontinuity. If it is negative, modes penetrate deeper in the planet; if positive, the sound speed jump acts as a wall where the propagation towards the planet is stopped.

\subsubsection{How to disentangle interior structure and EOS ?}

The previous chapter has put in evidence a major problem: how to discriminate between the influence of interior structure and that of the EOS? An answer is given by the seismological parameters. The work of Provost et al. (1993) has first shown the dependence between seismological parameters and interior structure parameters, and second proved that the seismological parameters are observable. The results are recalled by Fig. 22.17 and Fig. 22.18, and demonstrate that the measurement of asymptotic parameters corresponds to the measurement of interior structure parameters. 

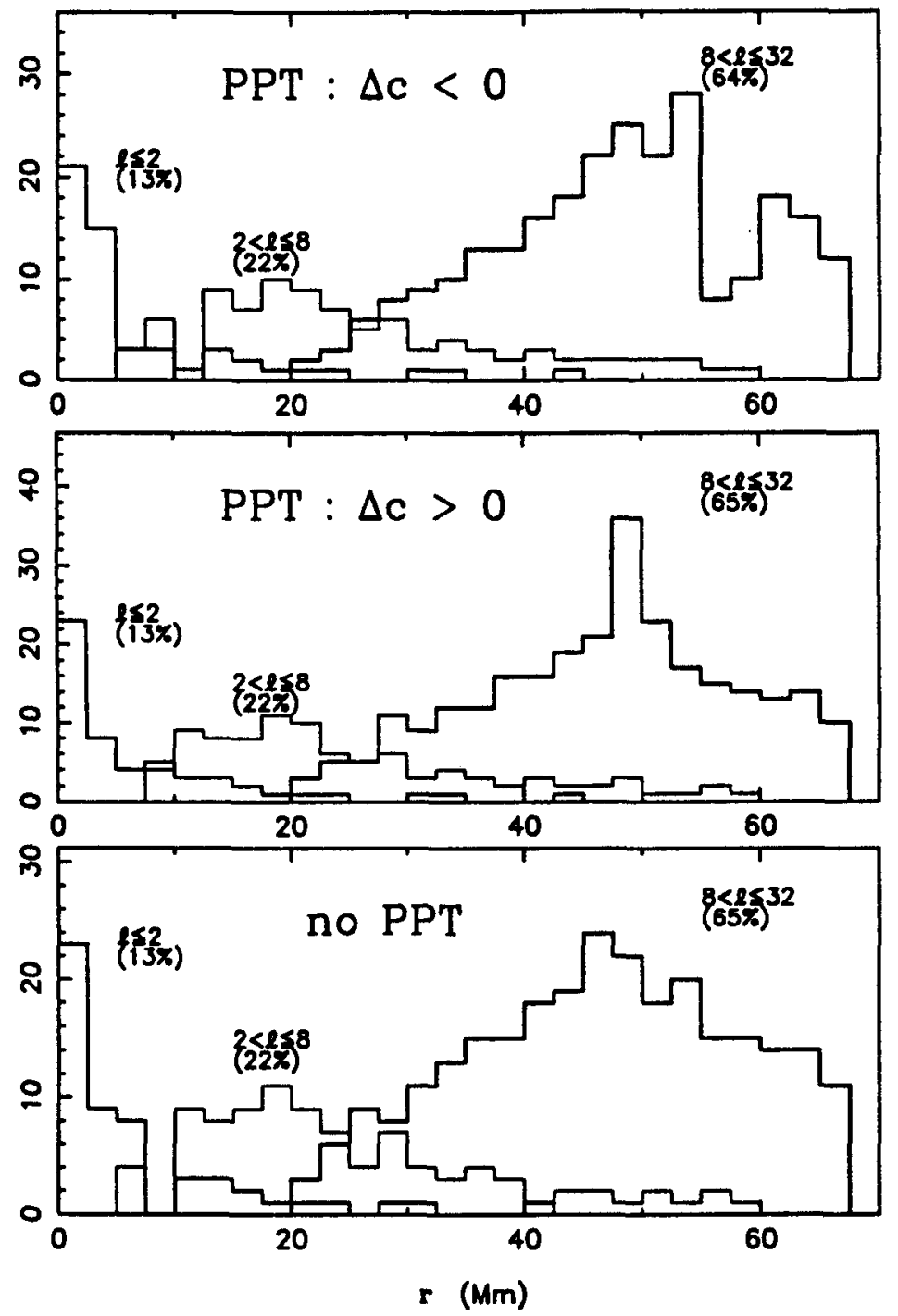

Fig. 22.16 Histograms counting the modes refracted at a given level in the planet, depending on the degree range. Three models have been used, which differ by the description of the PP'T. The number of high degree modes refracted at the PPT strongly depends on the sound speed discontinuity. If $\Delta c<0$, modes are refracted deeper in the planet; if $\Delta c>0$, total reflection may occur. The last model has no PPT.

\subsubsection{The future}

It appears now very important to define the next steps which must be done in dioseismology. The improvement of the description of the Jovian standard model is first required. The connection between the two fields - seismology and interior structure modelling - is already operative and fruitful. New 


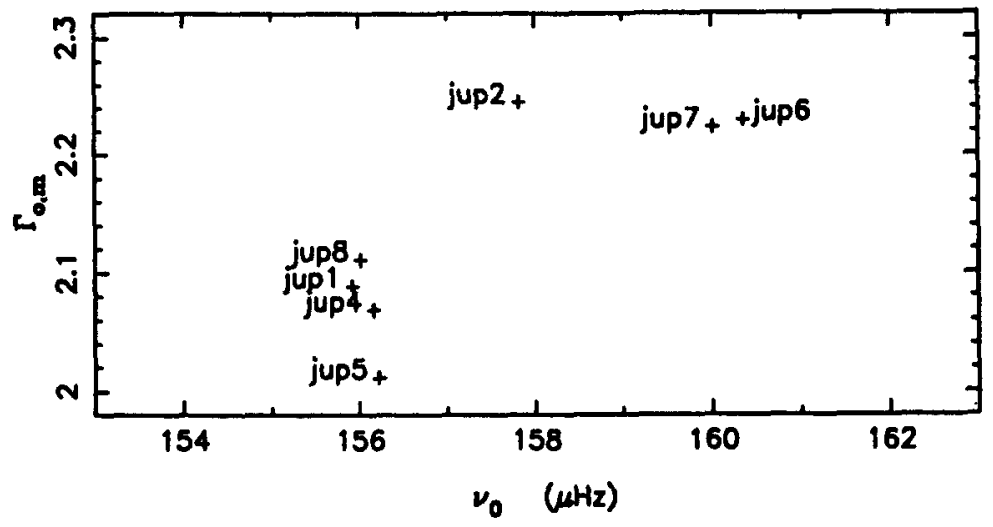

Fig. 22.17 $\left[\nu_{0}, \Gamma_{0, m}\right]$ diagram, indicating how the measure of the frequency $\nu_{0}$ can be related to the interior structure. $\Gamma_{o, m}$ represents the adiabatic coefficient which fits the inner (metallic) region of the fluid envelope. Models with a real PPT (JUP6 and JUP7) are separated from the others because of a higher $\nu_{0}$ value.

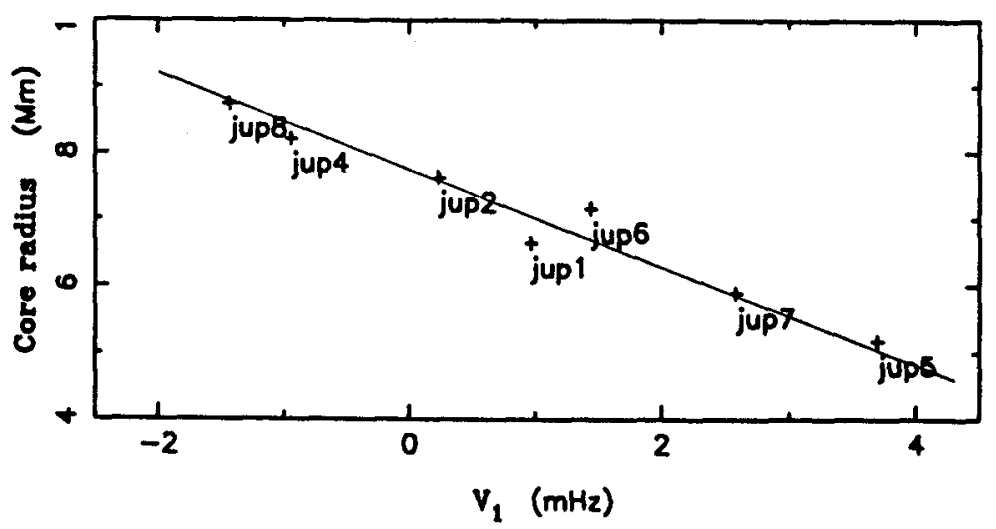

Fig. 22.18 $\left[V_{1}, r_{c}\right]$ diagram, where $r_{c}$ is the core radius. The quasi-linear dependence proves that the observation of the characteristic frequency $V_{1}$ provides the direct measurement of the core radius.

observations of Jovian oscillations are urgently needed. In order to improve the quality of the observed spectra, three solutions might be considered:

- The window effect can be reduced only with ground-based observations made with a network of site, or with space observation.

- In order to disentangle the spectrum, imaging must be developped (IR photometry at $10 \mu \mathrm{m})$. An image of the Jovian oscillation field permits the calculation of different spectra corresponding to different degree ranges and with a much lower mode density. 
- The cruise flight of an interplanetary spacecraft is a unique solution for observing continuously stellar or planetary oscillations. This solution will be used during the cruise flight of Mars94 (Baglin 1991), for asteroseismology.

Observing Saturn in the last years was far from favorable, with the ring system vignetting the major part of the Southern hemisphere. However, Saturn has been observed in July 1993 with the Fourier transform spectrometer at CFHT. The data reduction is under progress. During the opposition in August 1995, the rings will be seen edge on, providing a unique opportunity to obtain a regular and symmetric visibility of the modes.

\subsection{Conclusion}

In this paper, we have reviewed the first development of a new domain: the seismological study of giant planets, or dioseismology. Theoretical results, derived from helioseismology, are much in advance compared to the observations, except for the understanding of the modes excitation. The observations tend to prove the detection of the oscillations on Jupiter, with pretty large amplitudes, but they cannot yet be translated precisely in terms of interior structure. Even if theoretical results have shown the complexity of the oscillation spectrum due to the structure discontinuities, dioseismology is able to interpret their signature. One current problem is to distinguish between the different signatures. The one due to the core is obvious, and will provide information on the core size and structure. The direct observation of the PPT is more difficult, being hidden by the core. Finally, the new seismological constraints imply the development of a new generation of Jovian interior models.

\section{Acknowledgements}

I thank very much the organizers of the colloquium, first for having given the opportunity of a first review about dioseismolgy, second for having run the risk of inviting a novice in research. Je tiens également à remercier Daniel Gautier, pour ses directives toujours judicieuses ainsi que son aide précieuse.

This work has been supported by the Programme National de Planétologie from the Institut National des Sciences de l'Univers (INSU) and by the Groupement de Recherches 'Structure interne des étoiles et des planètes géantes' from the Centre National de la Recherche Scientifique (CNRS). 


\section{References}

Baglin A. 1991. Adv. Space Res., 11, 4, (4)133-(4)140.

Bercovici D. and G. Schubert 1987. Icarus 69, 557-565.

Campbell J. K. and S. P. Synnot 1985. Astron. J. 90, 364-372.

Chabrier G., D. Saumon, W.B. Hubbard and J.I. Lunine 1992. ApJ 391, 817-826.

Christensen-Dalsgaard J., T.L. Duvall Jr., D.O. Gough, J.W. Harvey, and E.J.

Rhodes 1985. Nature 315, 378-382.

Deming D., M. J. Mumma, F. Espenak, D. E. Jennings, Th. Kostiuk, G.

Wiedemann, R. Loewenstein, and J. Piscitelli 1989. ApJ 343, 456-467.

Gautier D. and T. Owen 1989. In Origin and Evolution of Planetary and

Satellites Atmospheres, (S.K. Atreya, J.B. Pollack and M.S. Matthews, Eds.)

University of Arizona Press, Tucson.

Guillot T., D. Gautier, Chabrier G., B.Mosser. Submitted to A\&A

Gelly B., G. Grec and E. Fossat 1986. A\&A 164, 383-394.

Goldreich P. and P. Kumar 1988. Astrophys. Journal 326, 462-478.

Gough D.O. 1986. In Hydrodynamics and MHD Problems in the Sun and Stars

(Y.Osaki, Ed.), 117-143. University of Tokyo Press.

Gudkova T.V., V.N. Zharkov and V.V. Leont'ev 1989. [Astron. Vestnik, 22,3

252-261], Solar Sys. Res 22, 159-166

Hubbard W.B. 1968. ApJ 152, 745-753.

Hubbard W.B. and M.S. Marley 1989. Icarus 78, 102-118.

Lee U. 1993. ApJ. 405, 359-374.

Lindal G.F., G.E. Wood, G.S. Levy, J.D. Anderson, D.N. Sweetnam, H.B. Hotz,

B.J. Buckles, D.P. Holmes, P.E. Doms, V.R. Eshelman, G.L. Tyler and T.A.

Croft 1981. J. Geophys. Res. 86, 8721-8727.

Marley M.S. 1991. Icarus 94, 420-435.

Mosser B., D. Gautier and Ph. Delache 1988. In Seismology of the Sun and Sun

like-stars. Tenerife, Spain, Sept. 1988. Proc. ESA, SP-286, 593-594.

Mosser B. 1990. Icarus 87, 198-209.

Mosser B., F.-X. Schmider, Ph. Delache and D. Gautier 1991. ApJ 251, 356-364.

Mosser B., D. Gautier and Th. Kostiuk 1992. Icarus 96, 15-26

Mosser B. 1992. Etude des oscillations globales de Jupiter et des planètes géantes. $\mathrm{PhD}$ thesis, Université Paris-XI, Orsay.

Mosser B., D. Mékarnia, J.-P. Maillard, J. Gay, D. Gautier and Ph. Delache 1993. $A \& A$ 267, 604-622.

Pottasch 1992. A\&A 264, 138.

Provost J., B. Mosser and G. Berthomieu 1993. A\&A 274, 595-611.

Saumon D. and G. Chabrier 1989. Phys. Rev. Letters 62, 2397-2400.

Saumon D., W.B. Hubbard, G. Chabrier and H.M. Van Horn 1992. ApJ 391, 827-831.

Schmider F.-X., B. Mosser and E. Fossat 1991. A\&A 248, 281-291.

Stevenson D.J. 1985. Icarus 62, 4-15.

Tassoul M. 1980. ApJ Suppl 43, 469-490.

Unno W. ,Y. Osaki, H. Ando and H. Shibashi 1979. Nonradial oscillation of stars, (W. Unno, Ed.), 149-159. University of Tokyo press.

Vorontsov S.V., V.N. Zharkov and V.M. Lubimov 1976. Icarus 27, 109-118.

Vorontsov S.V. and V.N. Zharkov 1981. Astron. Zh. 58, 1101-1114, [Sov. Astron. 25, 627-634, 1982].

Vorontsov S.V. 1981. Astron. Zh. 58, 1275-1285, [Sov. Astron. 25, 724-729, 1982].

Vorontsov S.V. 1984a. Astron. Zh. 61, 700-707, [Sov. Astron. 28, 410-414, 1984]. 
Vorontsov S.V. 1984b. Astron. Zh. 61, 854-859, [Sov. Astron. 28, 500-503, 1984].

Vorontsov S.V., T.V. Gudkova and V.N. Zharkov 1989. Pis. Astron. Zur. 15, 646-653.

Zharkov V.N. and V.P. Trubitsyn 1978. In Physics of the planetary interiors

(W.B. Hubbard, Ed.), 221-284. Pachart publishing house, Tucson.

Zharkov V.N. and T.V. Gudkova 1991. Ann. Geophysicae 9, 357-366.

Zharkov V.N. and T.V. Gudkova 1992. In High-Pressure research: Application to Earth and Planetary Sciences, Y. Syono, M.H. Manghnani Eds, 357-366. 